\title{
MicroRNA-34a directly targets high-mobility group box 1 and inhibits the cancer cell proliferation, migration and invasion in cutaneous squamous cell carcinoma
}

\author{
SHANSHAN LI ${ }^{1}, \mathrm{CHENGQUN} \mathrm{LUO}^{2}, \mathrm{JUN}_{\mathrm{ZHOU}}^{3}$ and YONG ZHANG
}

${ }^{1}$ Department of Plastic and Reconstructive Surgery, Plastic Surgery Hospital, Chinese Academy of Medical Sciences, Peking Union Medical College, Beijing 100144; Departments of ${ }^{2}$ Burns and ${ }^{3}$ Neurology, The Third Xiangya Hospital, Central South University, Changsha, Hunan 410013; ${ }^{4}$ Department of Cosmetic and Plastic Surgery, The Third People's Hospital of Huaihua, Huaihua, Hunan 418000, P.R. China

Received January 24, 2017; Accepted July 11, 2017

DOI: 10.3892/etm.2017.5245

\begin{abstract}
Cutaneous squamous cell carcinoma (CSCC) is the second most common type of skin cancer with increasing incidence. In recent years, several microRNAs (miRs) have been demonstrated to serve an oncogenic or tumor suppressive role in CSCC. However, the exact role of miR-34a in CSCC and the underlying regulatory mechanism remain unclear. The present study aimed to investigate the regulatory mechanism of miR-34a in the malignant phenotypes of CSCC cells using MTT assay, wound healing assay and transwell assay. It was observed that miR-34a was significantly downregulated in CSCC tissues and cell lines, and low miR-34a expression was associated with the aggressive progression of CSCC. Restoration of miR-34a significantly suppressed the proliferation, migration and invasion of CSCC SCL-1 cells. High-mobility group box 1 (HMGB1) was then identified as a target gene of miR-34a in SCL-1 cells using bioinformatics prediction. The expression of HMGB1 was significantly upregulated in the CSCC tissues and cell lines. Furthermore, the protein expression of HMGB1 was negatively regulated by miR-34a in SCL-1 cells, while overexpression of HMGB1 impaired the inhibitory effects of miR-34a on SCL-1 cells. These findings suggest that miR-34a represses the malignant phenotypes of CSCC cells, at least partly, via the inhibition of HMGB1. Therefore, miR-34a may be used as a promising therapeutic candidate for CSCC.
\end{abstract}

Correspondence to: Dr Yong Zhang, Department of Cosmetic and Plastic Surgery, The Third People's Hospital of Huaihua, 231 West Tianxing Road, Huaihua, Hunan 418000, P.R. China

E-mail: zhangyong0922@qq.com

Key words: cutaneous squamous cell carcinoma, microRNA-34a, high-mobility group box 1, proliferation, migration, invasion

\section{Introduction}

Cutaneous squamous cell carcinoma (CSCC) is one of the most common types of cancer affecting the skin, and is more aggressive in comparison with other skin carcinomas $(1,2)$. The incidence of CSCC has been increasing in recent years, and the prognosis of patients with advanced CSCC is unsatisfactory $(2,3)$. Therefore, the development of effective treatment strategies for CSCC is urgently required. Investigation on the molecular mechanism underlying CSCC growth and metastasis may help to identify potential therapeutic candidates and targets for CSCC.

MicroRNAs (miRs), a type of small noncoding RNAs with a length of 18-25 nucleotides, have been demonstrated to function as key regulators of gene expression via directly binding to the seed sequence in the 3'-untranslated region (3'UTR) of their target mRNAs, consequently causing translation repression or mRNA degradation $(4,5)$. Through regulating the protein expression of their target genes, miRs participate in various cellular biological processes, including cell proliferation, apoptosis, migration, invasion and tumorigenesis (6-8). In recent years, certain miRs have been implicated in the development and malignant progression of CSCC $(9,10)$. For instance, miR-365 serves a promoting role in CSCC through targeting nuclear factor I/B (9). In addition, miR-125b directly targets matrix metallopeptidase 13 and inhibits the CSCC cell proliferation, migration, and invasion (10). Among these miRs, miR-34a has been reported to be significantly downregulated in the skin and oral SCC tissues (11). However, the exact role of miR-34a in the regulation of CSCC cells remains unclear.

High-mobility group box 1 (HMGB1), a member of the HMGB superfamily, is a non-histone, nuclear DNA-binding protein that participates in the regulation of DNA organization and gene transcription $(12,13)$. It has been demonstrated that HMGB1 serves an oncogenic role in different types of human cancer, including CSCC (14). Sun et al (14) reported that HMGB1 promotes tumor metastasis in CSCC via the phosphoinositide 3-kinase/protein kinase $\mathrm{B}$ and mitogen-activated protein kinase signaling pathways. However, the regulatory 
mechanism underlying HMGB1 expression in CSCC remains unknown.

Therefore, the present study aimed to investigate the regulatory mechanism of miR-34a underlying CSCC growth and metastasis, as well as the involvement of HMGB1.

\section{Materials and methods}

Tissue collection. The present study was approved by the Ethics Committee of Plastic Surgery Hospital, Chinese Academy of Medical Sciences, Peking Union Medical College (Beijing, China). A total of 72 pairs of CSCC tissues and adjacent non-tumor tissues were collected from patients, subsequent to obtaining informed consents. The clinical information of the patients participating in the study is summarized in Table I. Following surgical resection, the tissues were immediately frozen in liquid nitrogen and stored at $-80^{\circ} \mathrm{C}$ until further use.

Cell culture. The human normal skin cell line HaCaT and the CSCC cell lines A431 and SCL-1 were purchased from the Cell Bank of Type Culture Collection, Chinese Academy of Sciences (Shanghai, China). Cells were cultured in Dulbecco's modified Eagle's medium (DMEM) with $10 \%$ fetal bovine serum (FBS; both from Thermo Fisher Scientific, Inc., Waltham, MA, USA) in a $37^{\circ} \mathrm{C}$ humidified atmosphere with $5 \% \mathrm{CO}_{2}$.

Cell transfection. Lipofectamine 2000 reagent (Thermo Fisher Scientific, Inc.) was used to perform cell transfection, in accordance with the manufacturer's protocol. Briefly, SCL-1 cells were transfected with the negative control scrambled miRNA (miR-NC), miR-34a mimic, miR-34a inhibitor, co-transfected with miR-34a mimic and blank pcDNA3.1 vector (miR-34a + blank), or co-transfected with miR-34a mimic and pcDNA3.1-HMGB1 open reading frame (ORF) plasmid (miR-34a + HMGB1), respectively. Transfection was for $48 \mathrm{~h}$ at $37^{\circ} \mathrm{C}$.

Reverse transcription-quantitative polymerase chain reaction (RT-qPCR) assay. Total RNA was extracted from the tissues and transfected cells using TRIzol reagent (Thermo Fisher Scientific, Inc.), according to the manufacturer's protocol. The RNA concentration was measured using Qubit 3.0 (Thermo Fisher Scientific, Inc.). Next, the total RNA was converted into cDNA using a High Capacity cDNA Reverse Transcription kit (Thermo Fisher Scientific, Inc.), according to the manufacturer's instructions. The miR expression was then examined by qPCR using a PrimeScript ${ }^{\circledR}$ miRNA RT-PCR kit (Takara Biotechnology Co., Ltd., Dalian, China) on an ABI 7500 thermocycler (Thermo Fisher Scientific, Inc.), with U6 used as an internal reference. The mRNA expression was determined using the standard SYBR-Green RT-PCR kit (Takara Biotechnology Co., Ltd.) on an ABI 7500 thermocycler, with GAPDH used as an internal reference. These procedures were performed according to the manufacturer's protocols. The primer sequences used in qPCR were as follows: HMGB1 forward, 5'-TATGGCAAAAGCGGACAAGG-3' and reverse, 5'-CTTCGCAACATCACCAATGGA-3'; GAPDH forward, 5'-ACA ACT TTGGTATCGTGGAAGG-3' and reverse, 5'-GCCATCACGCCACAGTTTC-3'. The primers for miR expression detection were purchased from Fulgene
(Guangzhou, China). The reaction was conducted at $95^{\circ} \mathrm{C}$ for $5 \mathrm{~min}$, followed by 40 cycles of denaturation at $95^{\circ} \mathrm{C}$ for $30 \mathrm{sec}$ and an annealing/elongation step at $60^{\circ} \mathrm{C}$ for $30 \mathrm{sec}$. The relative expression was analyzed by the $2^{-\Delta \Delta \mathrm{Cq}}$ method (15).

Western blot analysis. Cells were lysed with ice-cold radioimmunoprecipitation assay buffer (Thermo Fisher Scientific, Inc.). The concentration of protein was determined with a BCA Protein Assay kit (Thermo Fisher Scientific, Inc.), according to the manufacturer's protocol. Protein was separated by $10 \%$ SDS-PAGE and then transferred onto a polyvinylidene difluoride (PVDF) membrane (Thermo Fisher Scientific, Inc.). Next, the PVDF membrane was incubated with phosphate-buffered saline (PBS) containing 5\% non-fat milk (Yili Industrial Group Co., Ltd., Beijing, China) for $3 \mathrm{~h}$ at room temperature. Following washing with PBS for three times, the PVDF membrane was incubated with rabbit anti-human HMGB1 antibody (1:50; ab18256) or rabbit anti-human GAPDH antibody (1:100; ab9485) (both from Abcam, Cambridge, MA, USA) at $37^{\circ} \mathrm{C}$ for $3 \mathrm{~h}$. Subsequent to washing with PBS for three times, the PVDF membrane was then incubated with goat anti-rabbit IgG secondary antibody (1:5,000; ab7090; Abcam) at room temperature for $1 \mathrm{~h}$. A Chemiluminescent Substrate kit (Thermo Fisher Scientific, Inc.) was then used to detect the signals, according to the manufacturer's protocol. The relative protein expression was analyzed by Image-Pro Plus software (version 6.0; Media Cybernetics, Inc., Rockville, MD, USA). GAPDH was used as the internal reference.

Cell proliferation analysis. SCL-1 cells $\left(2 \times 10^{4}\right.$ cells $\left./ \mathrm{ml}\right)$ were seeded into 96 -well plates (5,000 cells/well) and incubated at $37^{\circ} \mathrm{C}$ for 24,48 and $72 \mathrm{~h}$. At each time point, $0.5 \% \mathrm{MTT}$ solution was added, and the cells were then incubated at $37^{\circ} \mathrm{C}$ for $4 \mathrm{~h}$. Subsequently, cells were centrifuged at 1,500 x g for $3 \mathrm{~min}$ at room temperature, the cell supernatant was discarded, and $150 \mu$ l dimethyl sulfoxide was added to dissolve the formazan. The optical density was then measured using a microplate reader (Bio-Rad Laboratories, Inc., Hercules, CA, USA) at a wavelength of $570 \mathrm{~nm}$.

Cell migration analysis. SCL-1 cells were cultured to full confluence in 6-well plates $(500,000$ cells/well), and then a plastic scraper was used to create wounds ( 1 $\mathrm{mm}$ in width). Subsequently, the cells were washed with PBS and then incubated in DMEM containing $10 \% \mathrm{FBS}$ at $37^{\circ} \mathrm{C}$ for $24 \mathrm{~h}$. Images of the cells were captured under a light microscope and the number of cells migrating into the scratched area was recorded.

Cell invasion analysis. Cell invasion was determined using transwell chambers pre-coated with Matrigel (BD Biosciences, Franklin Lakes, NJ, USA), according to the manufacturer's protocol. Briefly, SCL-1 cell suspension $\left(1 \times 10^{6}\right.$ cells $\left./ \mathrm{ml}\right)$ was prepared in DMEM. Next, $300 \mu$ l of the SCL-1 cell suspension was added into the upper chamber, while $300 \mu$ DMEM with $10 \%$ FBS was added into the lower chamber. Following incubation at $37^{\circ} \mathrm{C}$ for $24 \mathrm{~h}$, cells that had not migrated through the pores were wiped out using a cotton-tipped swab. The filters were then fixed in $90 \%$ alcohol at room temperature for $10 \mathrm{~min}$, and stained by crystal violet (Sigma-Aldrich; Merck, Darmstadt, Germany). Images of the invading cells 
Table I. Association between miR-34a expression and clinicopathological characteristics of cutaneous squamous cell carcinoma patients.

\begin{tabular}{|c|c|c|c|c|}
\hline \multirow[b]{2}{*}{ Variables } & \multirow[b]{2}{*}{ No. of cases $(n=72)$} & \multicolumn{2}{|c|}{ miR-34a expression } & \multirow[b]{2}{*}{ P-value } \\
\hline & & Low $(n=39)$ & $\operatorname{High}(\mathrm{n}=33)$ & \\
\hline Age (years) & & & & 0.813 \\
\hline$<60$ & 33 & 17 & 16 & \\
\hline$\geq 60$ & 39 & 22 & 17 & \\
\hline Sex & & & & 0.154 \\
\hline Male & 40 & 25 & 15 & \\
\hline Female & 32 & 14 & 18 & \\
\hline Histopathological grade & & & & $0.016^{\mathrm{a}}$ \\
\hline Well to moderate & 43 & 18 & 25 & \\
\hline Poor & 29 & 21 & 8 & \\
\hline Lymph node metastasis & & & & $0.012^{\mathrm{a}}$ \\
\hline Present & 25 & & 6 & \\
\hline Absent & 47 & & 27 & \\
\hline
\end{tabular}

were obtained under an inverted microscope (Olympus Corp., Tokyo, Japan). The invading cells were counted.

Bioinformatics prediction. TargetScan version 7.1 online software (www.targetscan.org) was used to predict the potential targets of miR-34a, according to the manufacturer's instructions. Briefly, 'human' was selected as the target species, and 'miR-34a' was inserted as the investigated miR. MiR-34a was predicted to be able to directly bind to the seeding sequences of the 3'-UTR of HMGB1.

Luciferase reporter gene assay. The wild type (WT) and mutant type (MT) of HMGB1 3'UTR luciferase reporter gene plasmids were generated by Yearthbio (Changsha, China). The SCL-1 cells were then co-transfected with miR-34a mimic or miR-NC, and with the WT- or MT-HMGB1-3'UTR reporter plasmid using Lipofectamine 2000 reagent. Subsequent to incubation at $37^{\circ} \mathrm{C}$ for $48 \mathrm{~h}$, the luciferase activity was examined using a Dual-Luciferase Reporter Assay system (Promega Corp., Madison, WI, USA), according to the manufacturer's protocol.

Statistical analysis. Data are expressed as the mean value \pm standard deviation. SPSS version 19.0 software (IBM Corp., Armonk, NY, USA) was used to perform statistical analysis. The contingency data was analyzed by $\chi^{2}$ test. Data were also analyzed using Student's t-test for two-group comparisons or one-way analysis of variance for comparison of more than two groups. A P-value of $<0.05$ was considered to indicate a difference that was statistically significant.

\section{Results}

miR-34a is downregulated in CSCC tissues and cell lines. The miR-34a levels were examined using RT-qPCR in CSCC and adjacent non-tumor tissues. The results demonstrated that the expression levels of miR-34a were significantly lower in CSCC tissues compared with the adjacent non-tumor tissues (Fig. 1A). In addition, it was observed that miR-34a was significantly downregulated in CSCC cell lines, including A431 and SCL-1, when compared with the expression in normal skin HaCaT cells (Fig. 1B).

Furthermore, the clinical significance of miR-34a expression in CSCC was examined based on the correlation of this expression with the clinicopathological characteristics of patients. These CSCC patients were divided into low miR-34a expression group and high miR-34a expression group, based on the mean value (1.64) of miR-34a expression levels as the cut-off value. No significant association was detected between miR-34a expression and the age or gender of patients (Table I). However, the low miR-34a expression group had more CSCC patients with poor differentiation degree and lymph node metastasis, when compared with the high miR-34a expression group, indicating that low expression of miR-34a was found to be significantly associated with poor differentiation and presence of node metastasis (Table I). Based on these findings, it is suggested that miR-34a downregulation may contribute to the malignant progression of CSCC.

Restoration of miR-34a expression inhibits SCL-1 cell proliferation, migration and invasion. In order to restore the expression levels of miR-34a in the CSCC cells, SCL-1 cells were transfected with an miR-34a mimic. Following transfection, the miR-34a levels were significantly increased in the miR-34a group compared with the miR-NC group (Fig. 2A). In addition, the results of MTT, wound healing and transwell assays further demonstrated that the proliferation, migration and invasion of SCL-1 cells, respectively, were significantly reduced in the miR-34a group compared with the miR-NC group (Fig. 2B-D). These findings suggest that miR-34a exerted suppressive effects on the malignant phenotypes of SCL-1 cells. 
A

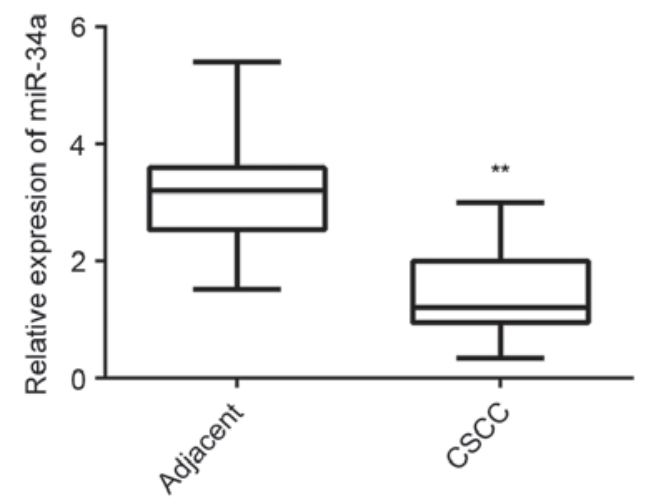

B

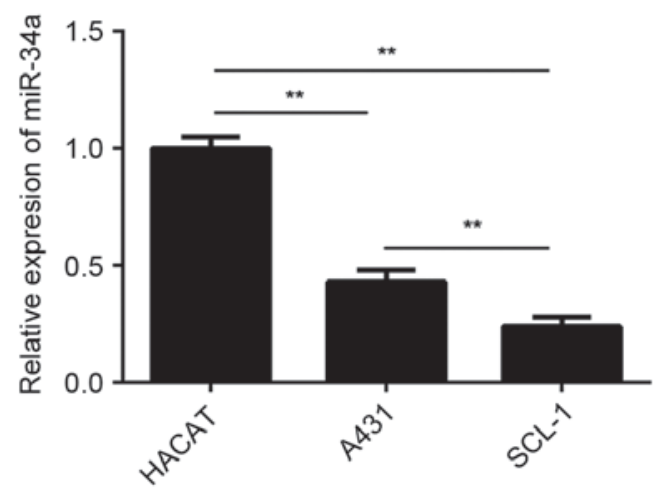

Figure 1. Reverse transcription-quantitative polymerase chain reaction was used to examine the expression levels of miR-34a in (A) CSCC tissues and their matched adjacent non-tumor tissues $\left({ }^{* *} \mathrm{P}<0.01\right.$ vs. adjacent non-tumor tissues) and (B) human CSCC cell lines (A431 and SCL-1) and human normal skin HaCaT cells $\left({ }^{* *} \mathrm{P}<0.01\right)$. miR, microRNA; CSCC, cutaneous squamous cell carcinoma.

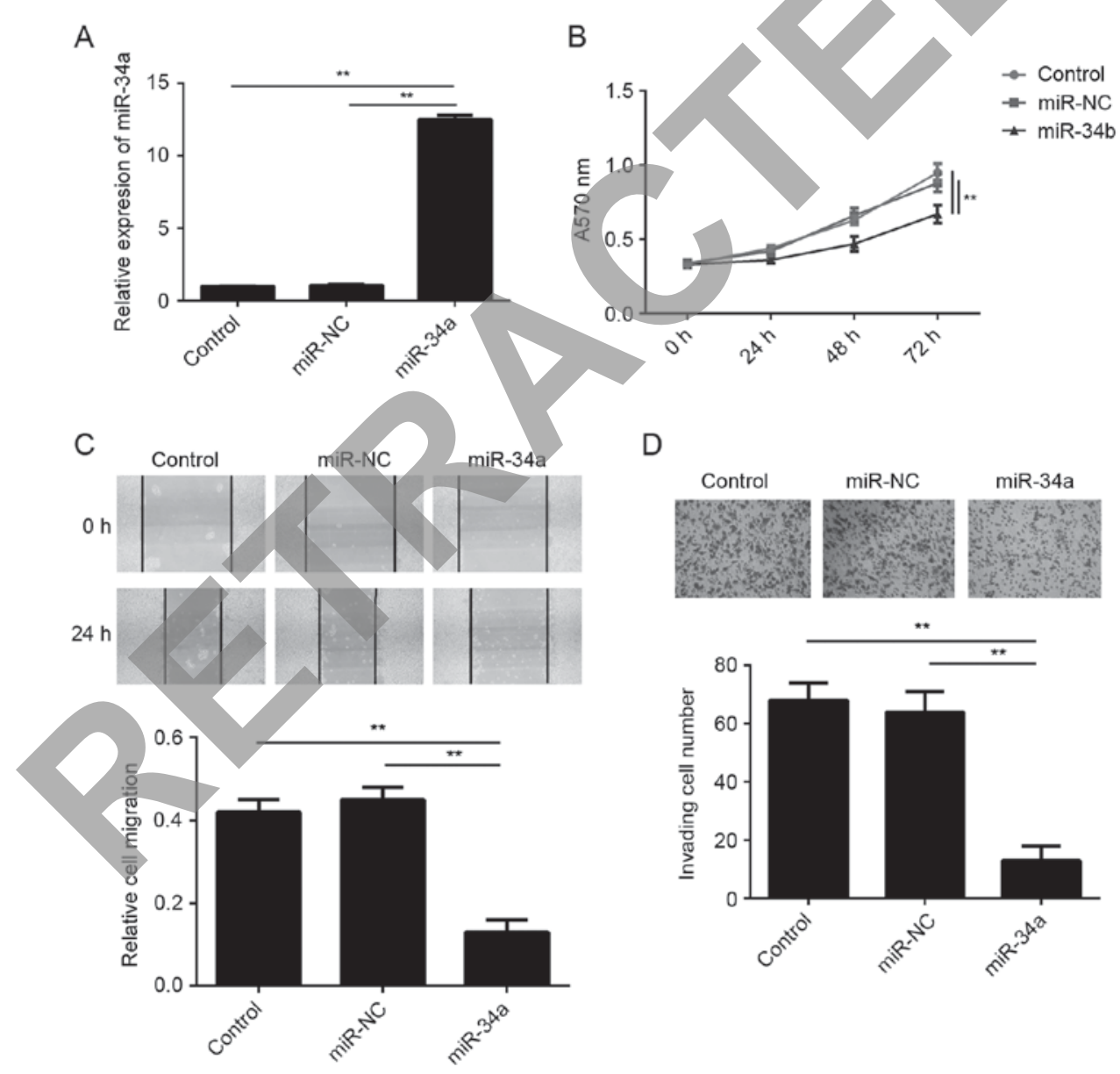

Figure 2. SCL-1 cells were transfected with miR-NC or miR-34a mimic. (A) Reverse transcription-quantitative polymerase chain reaction was used to examine the expression of miR-34a. (B) MTT, (C) wound healing and (D) Transwell assays were used to examine the cell proliferation, migration and invasion, respectively. Non-transfected cells were used as the control group. ${ }^{* *} \mathrm{P}<0.01$. miR-NC, scrambled negative control miR; miR, microRNA.

HMGBI is identified as a novel target gene of miR-34a in $S C L-1$ cells. The study then examined the putative target of miR-34a in CSCC cells using bioinformatics analysis. As shown in Fig. 3A, bioinformatics prediction results showed that miR-34a was able to directly bind to the seeding sequences of the 3'UTR of HMGB1. Subsequently, the WT and MT HMGB1 3'-UTR reporter gene plasmids were generated (Fig. 3B). A luciferase reporter gene assay was then performed to confirm whether HMGB1 was a target gene of miR-34a. As shown in Fig. 3C, the luciferase activity was significantly decreased in SCL-1 cells co-transfected with miR-34a mimic and WT-HMGB1-3'UTR reporter gene plasmid, when compared with the control group; however, no significant difference was observed upon co-transfection with 


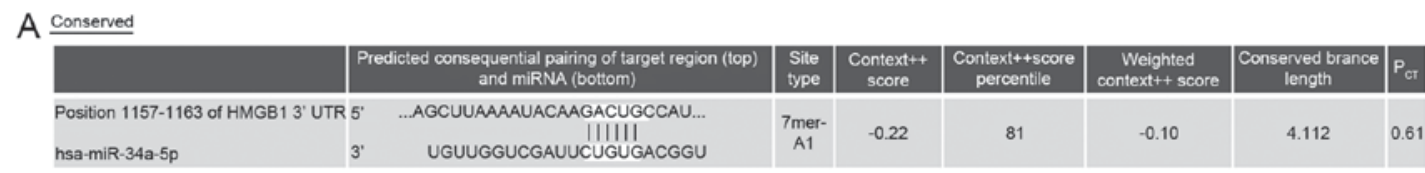

B

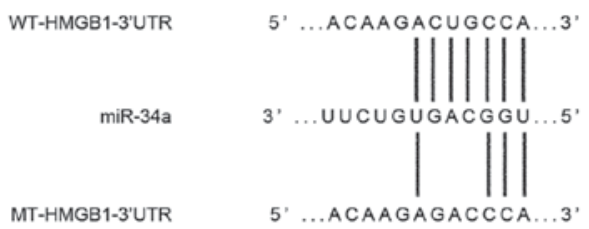

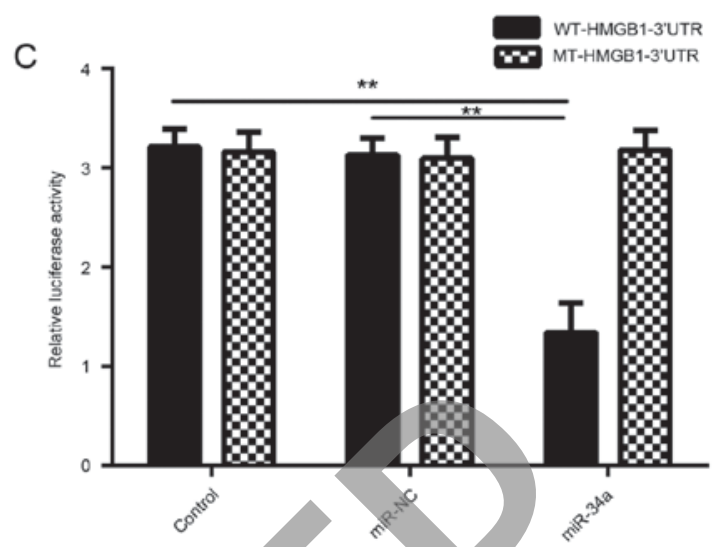

Figure 3. (A) Bioinformatics analysis demonstrated the putative seed region of miR-34a in the 3-UTR of HMGB1. (B) Generation of the WT and MT HMGB1 3'-UTR reporter gene plasmids. (C) Luciferase reporter gene assay was conducted in SCL-1 cells co-transfected with miR-34a mimic and miR-NC. ${ }^{* *} \mathrm{P}<0.01$. WT, wild-type; MT, mutated-type; 3'UTR, 3'-untranslated region; HMGB1, high-mobility group box 1; miR-NC, scrambled negative control miR; miR, microRNA.

miR-34a mimic and the MT-HMGB1-3'UTR reporter gene plasmid. These data indicate that HMGB1 is indeed a target gene of miR-34a.

As miRs generally function as a negative regulator of the expression of their target genes, the present study then investigated the effects of miR-34a on the expression of HMGB1 in SCL-1 cells. The results indicated that the mRNA and protein expression levels of HMGB1 were significantly lower in the miR-34a group compared with the miR-NC group (Fig. 4A and B). To further confirm these findings, SCL-1 cells were transfected with miR-34a inhibitor or with NC inhibitor, serving as the control group. Following transfection, the miR-34a levels were significantly reduced (Fig. 4C). Furthermore, it was observed that the mRNA and protein expression levels of HMGB1 were significant higher in the miR-34a inhibitor group compared with the NC inhibitor group (Fig. 4D and E). These aforementioned data demonstrated that miR-34a negatively regulated the expression of HMGB1 by directly bind to its mRNA 3'UTR.

HMGB1 is upregulated in CSCC tissues and cell lines. The expression of HMGB1 was further examined in the CSCC tissues and cell lines. As indicated in Fig. 5A, the mRNA levels of HMGB1 were significantly higher in the CSCC tissues compared with the adjacent non-tumor tissues. The mRNA and protein levels of HMGB1 were also increased in the examined CSCC cell lines (A431 and SCL-1) compared with the normal $\mathrm{HaCaT}$ cells (Fig. 5B and C). Based on these data, it is suggested that upregulation of HMGB1 may be involved in the CSCC development and progression.

Overexpression of HMGB1 impairs the suppressive effects of miR-34a on SCL-1 cells. Based on the aforementioned data, it was speculated that HMGB1 may be involved in the miR-34a-mediated proliferation, migration and invasion of SCL-1 cells. To clarify this speculation,
miR-34a-overexpressing SCL-1 cells were transfected with pcDNA3.1-HMGB1 ORF plasmid or with blank pcDNA3.1 vector, serving as the control. As shown in Fig. 6A and B, the mRNA and protein levels of HMGB1 were significantly increased in the miR-34a + HMGB1 group, when compared with the miR-34a + blank group. The MTT, wound healing and transwell assays further indicated that the proliferation, migration and invasion of SCL-1 cells were increased in the miR-34a + HMGB1 group compared with the miR-34a + blank group (Fig. 6C-E). These findings suggest that miR-34a serves a suppressive role in the regulation of SCL-1 cell proliferation, migration and invasion, at least partly, through targeting miR-34a.

\section{Discussion}

The regulatory mechanism of miR-34a in CSCC remains largely unclear. In the present study, it was revealed that miR-34a was significantly downregulated in CSCC tissues and cell lines, while low miR-34a expression was associated with the aggressive progression of CSCC. Restoration of the reduced miR-34a significantly suppressed the proliferation, migration and invasion of CSCC SCL-1 cells. HMGB1 was subsequently identified as a target gene of miR-34a in SCL-1 cells, and its expression was significantly upregulated in CSCC tissues and cell lines. Furthermore, the protein expression of HMGB1 was negatively regulated by miR-34a in SCL-1 cells, and overexpression of HMGB1 impaired the inhibitory effects of miR-34a on SCL-1 cells.

miR-34a has been demonstrated to serve tumor promoting or suppressive roles in different types of human cancer (16-18). For instance, Ma et al (16) reported that miR-34a inhibited the proliferation and promoted the apoptosis of non-small cell lung cancer cells by targeting transforming growth factor $\beta$ receptor 2. Besides, miR-34a inhibited osteosarcoma cell proliferation by reducing the expression of ether-à-go-go 1 (17). 
A

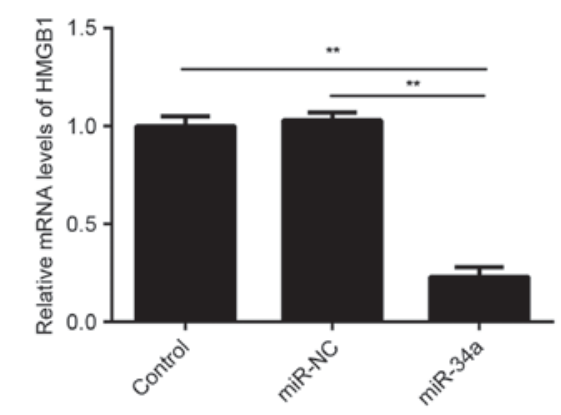

C

$E$
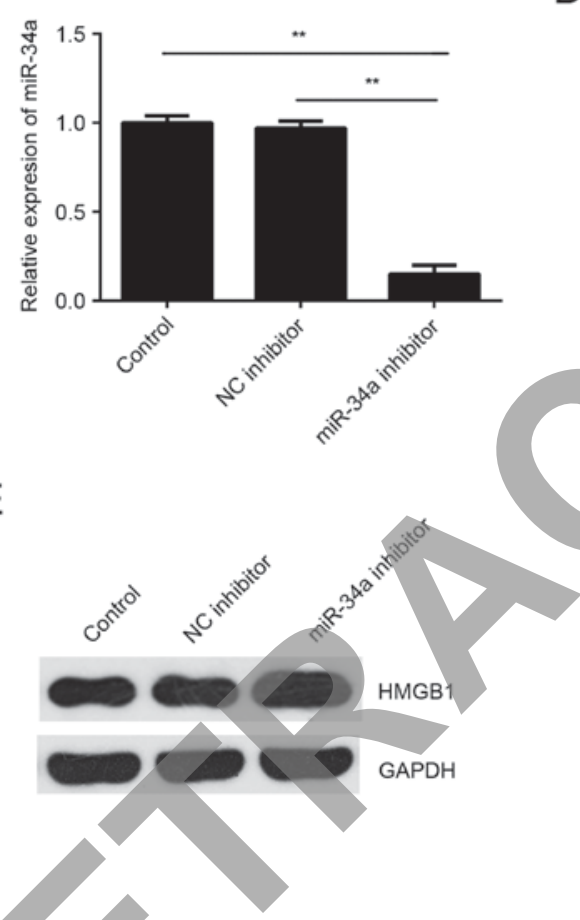

B

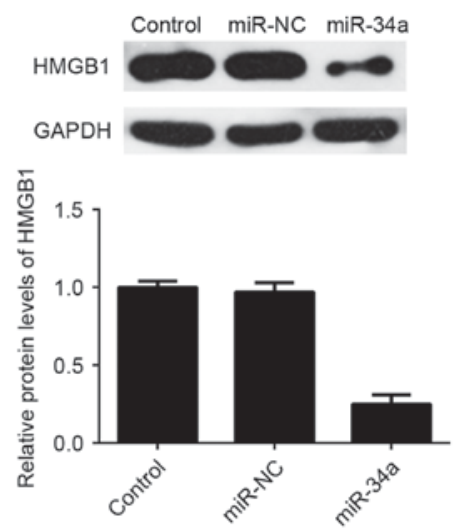

D

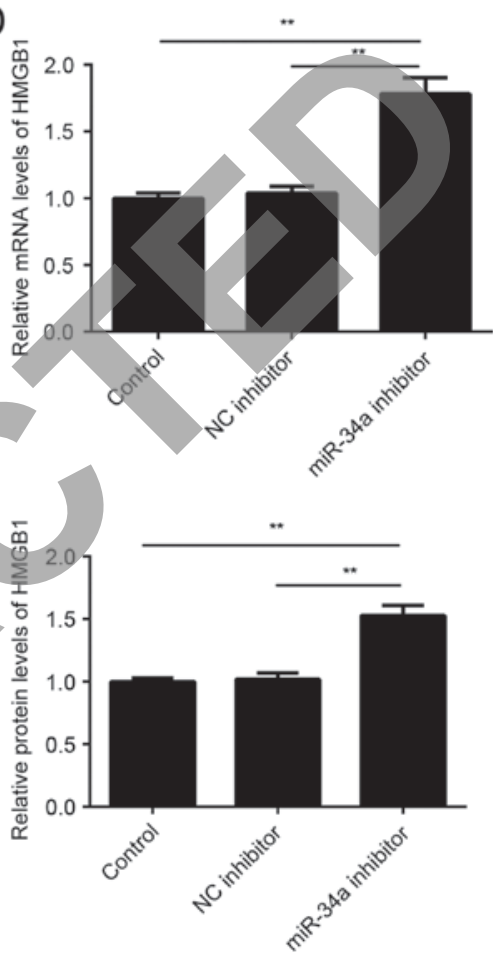

Figure 4. (A) RT-qPCR and (B) western blot analysis were conducted to examine the mRNA and protein expression levels, respectively, of HMGB1 in SCL-1 cells transfected with miR-NC or miR-34a mimic. (C) RT-qPCR was conducted to examine the miR-34a expression in SCL-1 cells transfected with miR-34a inhibitor or NC inhibitor, respectively. (D) RT-qPCR and (E) western blot analysis were conducted to examine the mRNA and protein expression levels of HMGB1, respectively. Non-transfected cells were used as the control group. ${ }^{* *} \mathrm{P}<0.01$. RT-qPCR, reverse transcription-quantitative polymerase chain reaction; miR-NC, scrambled miR; miR, microRNA; NC, negative control.

By contrast, upregulation of miR-34a has been implicated in invasive cervical cancer (18). Recently, Dotto and Karine (11) reported that miR-34a was significantly downregulated in skin and oral SCC tissues. In the present study, it was also observed that miR-34a was significantly downregulated in CSCC tissues and cell lines, when compared with the adjacent non-tumor tissues and normal skin cells, respectively. The results further demonstrated that the reduced expression of miR-34a was significantly associated with advanced clinical stage and lymph node metastasis. These findings suggest that downregulation of miR-34a is implicated in CSCC progression. Further investigation revealed that restoration of the miR-34a expression significantly inhibited the proliferation, migration and invasion of CSCC cells, suggesting that miR-34a may has suppressive effects on the CSCC growth and metastasis.

As miRs generally function through the inhibition of the expression of their targets (19), the potential target genes of
miR-34a were subsequently analyzed in the present study using bioinformatics prediction, and HMGB1 was predicted to be a target gene of miR-34a. Recently, miR-34a was found to suppress the proliferation, migration and invasion of human cervical and colorectal cancer cells via downregulation of HMGB1 (20). However, the association between miR-34a and HMGB1 in other types of human cancer, including CSCC, has not been previously reported, to the best of our knowledge. HMGB1 is generally upregulated in human cancer and functions as an oncogene $(21,22)$. For instance, Pang et al (21) reported that HMGB1 was significantly upregulated in cervical carcinoma, and promoted cell invasion and migration in vitro. Chen et al (22) revealed that HMGB1 promoted hepatocellular carcinoma progression through miR-21-mediated matrix metalloproteinase activity. To verify the association between miR-34a and HMGB1 in CSCC, a luciferase reporter gene assay was conducted in the current study, and the data 
A

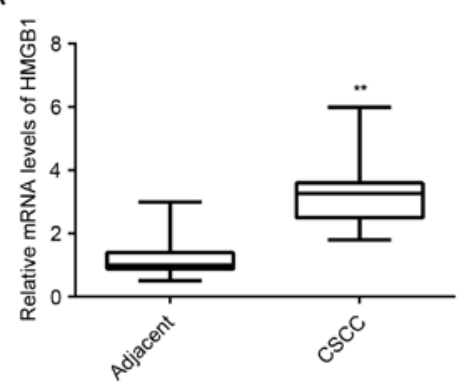

C

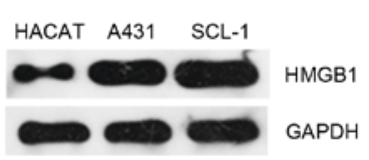

B
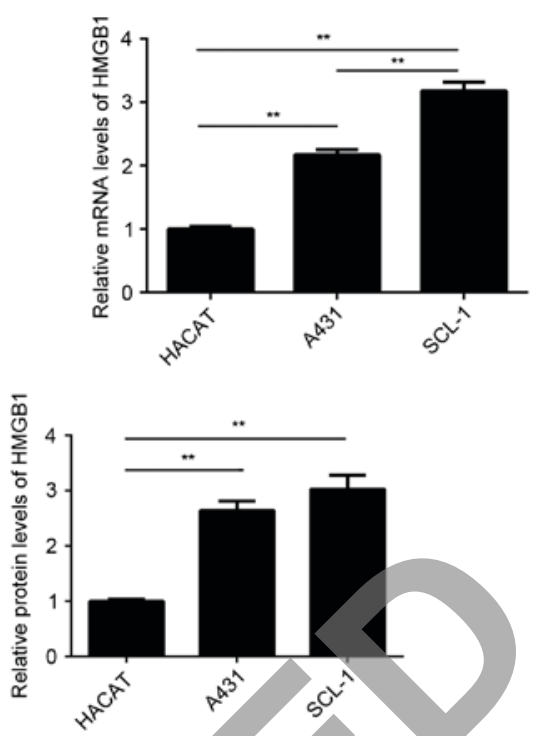

Figure 5. (A) RT-qPCR was used to examine the mRNA expression of HMGB1 in CSCC tissues and matched adjacent non-tumor tissues. "* P<0.01 vs. adjacent tissues. (B) RT-qPCR and (C) western blot analysis were used to examine the mRNA and protein expression levels, respectively, of HMGB1 in human CSCC cell lines (A431 and SCL-1) and human normal skin HaCaT cells. ${ }^{* *} \mathrm{P}<0.01$. HMGB1, high-mobility group box 1; RT-qPCR, reverse transcription-quantitative polymerase chain reaction; CSCC, cutaneous squamous cell carcinoma.

A
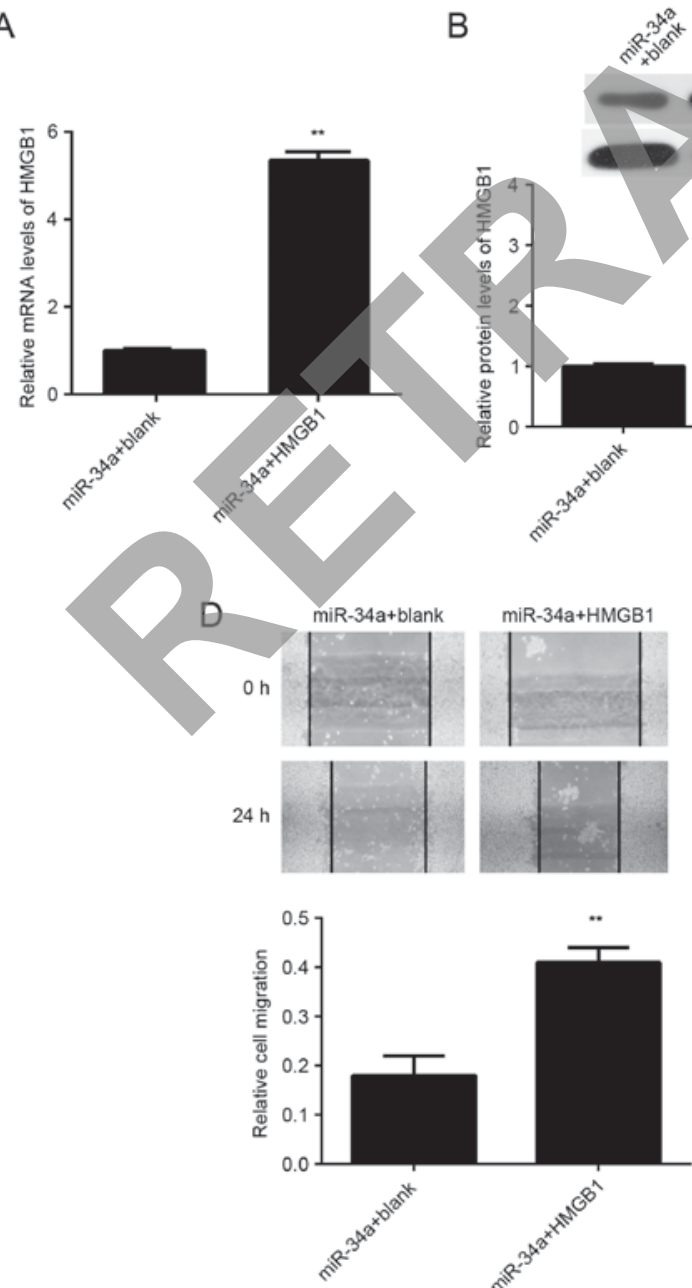

C

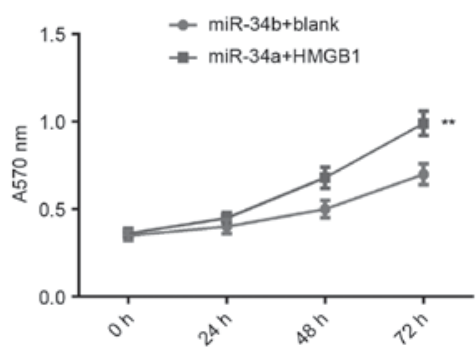

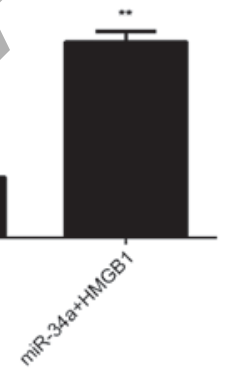

E

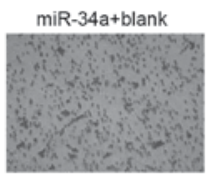

miR-34a+HMGB1
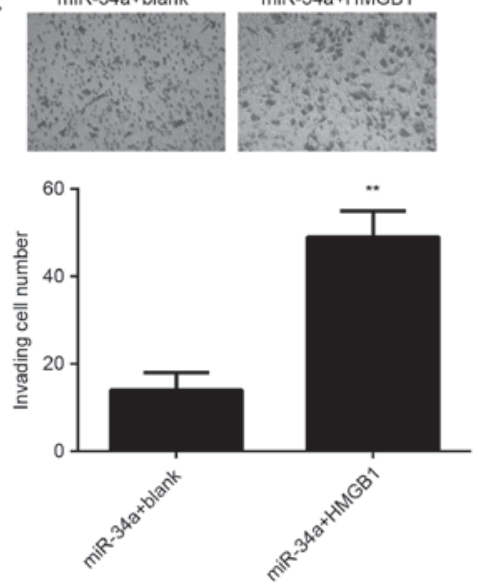

Figure 6. miR-34a-overexpressing SCL-1 cells were transfected with pcDNA3.1-HMGB1 open reading frame plasmid or blank pcDNA.31 vector. (A) Reverse transcription-quantitative polymerase chain reaction and (B) western blot analysis were conducted to detect the mRNA and protein expression levels of HMGB1, respectively. (C) MTT, (D) wound healing and (E) Transwell assays were used to examine the cell proliferation, migration and invasion, respectively. ${ }^{* * *} \mathrm{P}<0.01$ vs. miR-34a + blank group. miR, microRNA; HMGB1, high-mobility group box 1 . 
confirmed that HMGB1 was indeed a target gene of miR-34a in CSCC cells. Recently, Sun et al (14) reported that the levels of HMGB1 were higher in the CSCC cell supernatant compared with the human epidermoid carcinoma A431 cell supernatant. In the present study, HMGB1 was observed to be upregulated in CSCC tissues and cell lines, when compared with the adjacent non-tumor tissues and normal skin cells, respectively. Furthermore, overexpression of miR-34a caused a decrease in HMGB1 expression, while knockdown of miR-34a increased the HMGB1 expression in CSCC cells. Therefore, the upregulation of HMGB1 in CSCC may be due to the reduced expression of miR-34a.

Based on the aforementioned data, it was speculated that HMGB1 was involved in the suppressive effects of miR-34a on CSCC cells. Further investigation revealed that overexpression of HMGB1 impaired the suppressive effects of miR-34a on the CSCC cell proliferation, migration and invasion, which confirms this speculation. In addition, certain other miRs have also been demonstrated to directly target HMGB1, including miR-142 (23), miR-126 (24), miR-181 (25), miR-218 (26), miR-129 (27), miR-24 (28) and miR-141 (29). Therefore, the findings of the present study further expand the understanding of the regulatory mechanism of miRs/HMGB1 signaling in human cancer.

In conclusion, the present study demonstrated for the first time that miR-34a is downregulated in CSCC, and that it exerts suppressive effects on the proliferation, migration and invasion of CSCC cells, at least partly, via targeting HMGB1. Therefore, the present study suggests that miR-34a may be a potential therapeutic candidate for the treatment of CSCC.

\section{References}

1. Skulsky SL, O'Sullivan B, McArdle O, Leader M, Roche M, Conlon PJ and O'Neill JP: Review of high-risk features of cutaneous squamous cell carcinoma and discrepancies between the American Joint Committee on Cancer and NCCN clinical practice guidelines in oncology. Head Neck 39: 578-594, 2017.

2. Cheng J and Yan S: Prognostic variables in high-risk cutaneous squamous cell carcinoma: A review. J Cutan Pathol 43: 994-1004, 2016.

3. Sherwood V and Leigh IM: WNT signaling in cutaneous squamous cell carcinoma: A future treatment strategy? J Invest Dermatol 136: 1760-1767, 2016.

4. Zhu K, He Y, Xia C, Yan J, Hou J, Kong D, Yang Y and Zheng G: MicroRNA-15a inhibits proliferation and induces apoptosis in CNE1 nasopharyngeal carcinoma cells. Oncol Res 24: 145-151, 2016.

5. Wang G, Fu Y, Liu G, Ye Y and Zhang X: miR-218 inhibits proliferation, migration, and EMT of gastric cancer cells by targeting WASF3. Oncol Res 25: 355-364, 2017.

6. Lv H, Zhang Z, Wang Y, Li C, Gong W and Wang X: MicroRNA-92a promotes colorectal cancer cell growth and migration by inhibiting KLF4. Oncol Res 23: 283-290, 2016.

7. Liu X, Li J, Yu Z, Sun R and Kan Q: MiR-935 promotes liver cancer cell proliferation and migration by targeting SOX7. Oncol Res 25: 427-435, 2017.

8. Ji S, Zhang B, Kong Y, Ma F and Hua Y: MiR-326 inhibits gastric cancer cell growth through down regulating NOB1. Oncol Res 25: 853-861, 2017.

9. Zhou M, Zhou L, Zheng L, Guo L, Wang Y, Liu H, Ou C and Ding Z: miR-365 promotes cutaneous squamous cell carcinoma (CSCC) through targeting nuclear factor I/B (NFIB). PLoS One 9: e100620, 2014.
10. Xu N, Zhang L, Meisgen F, Harada M, Heilborn J, Homey B, Grandér D, Ståhle M, Sonkoly E and Pivarcsi A: MicroRNA-125b down-regulates matrix metallopeptidase 13 and inhibits cutaneous squamous cell carcinoma cell proliferation, migration, and invasion. J Biol Chem 287: 29899-29908, 2012.

11. Dotto GP and Karine L: miR-34a/SIRT6 in squamous differentiation and cancer. Cell Cycle 13: 1055-1056, 2014.

12. Ding J, Cui X and Liu Q: Emerging role of HMGB1 in lung diseases: Friend or foe. J Cell Mol Med 21: 1046-1057, 2017.

13. Anggayasti WL, Mancera RL, Bottomley S and Helmerhorst E: The self-association of HMGB1 and its possible role in the binding to DNA and cell-membrane receptors. FEBS Lett 591: 282-294, 2017.

14. Sun Y, Tu Y, He LI, Ji C and Cheng BO: High mobility group box 1 regulates tumor metastasis in cutaneous squamous cell carcinoma via the PI3K/AKT and MAPK signaling pathways. Oncol Lett 11: 59-62, 2016.

15. Livak KJ and Schmittgen TD: Analysis of relative gene expression data using real-time quantitative PCR and the 2(-Delta Delta C(T)) method. Methods 25: 402-408, 2001.

16. Ma ZL, Hou PP, Li YL, Wang DT, Yuan TW, Wei JL, Zhao BT, Lou JT, Zhao XT, Jin Y and Jin YX: MicroRNA-34a inhibits the proliferation and promotes the apoptosis of non-small cell lung cancer H1299 cell line by targeting TGF $\beta$ R2. Tumour Biol 36: 2481-2490, 2015.

17. Wu X, Zhong D, Gao Q, Zhai W, Ding Z and Wu J: MicroRNA-34a inhibits human osteosarcoma proliferation by downregulating ether à go-go 1 expression. Int J Med Sci 10: 676-682, 2013.

18. Ribeiro J, Marinho-Dias J, Monteiro P, Loureiro J, Baldaque I, Medeiros R and Sousa H: miR-34a and miR-125b expression in HPV infection and cervical cancer development. Biomed Res Int 2015: 304584, 2015.

19. Ambros V: The functions of animal microRNAs. Nature 431: 350-355, 2004.

20. Chandrasekaran KS, Sathyanarayanan A and Karunagaran D: Downregulation of HMGB1 by miR-34a is sufficient to suppress proliferation, migration and invasion of human cervical and colorectal cancer cells. Tumour Biol 37: 13155-13166, 2016.

21. Pang X, Zhang Y and Zhang S: High-mobility group box 1 is overexpressed in cervical carcinoma and promotes cell invasion and migration in vitro. Oncol Rep 37: 831-840, 2017.

22. Chen M, Liu Y, Varley P, Chang Y, He XX, Huang H, Tang D, Lotze MT, Lin J and Tsung A: High-mobility group box 1 promotes hepatocellular carcinoma progression through miR-21-mediated matrix metalloproteinase activity. Cancer Res 75: 1645-1656, 2015.

23. Wang Y, Ouyang M, Wang Q and Jian Z: MicroRNA-142-3p inhibits hypoxia/reoxygenation-induced apoptosis and fibrosis of cardiomyocytes by targeting high mobility group box 1 . Int J Mol Med 38: 1377-1386, 2016.

24. Tang ST, Wang F, Shao M, Wang Y and Zhu HQ: MicroRNA-126 suppresses inflammation in endothelial cells under hyperglycemic condition by targeting HMGB1. Vascul Pharmacol 88: 48-55, 2017

25. Liu Y, Hu X, Xia D and Zhang S: MicroRNA-181b is downregulated in non-small cell lung cancer and inhibits cell motility by directly targeting HMGB1. Oncol Lett 12: 4181-4186, 2016.

26. Gu J, Xu R, Li Y, Zhang J and Wang S: MicroRNA-218 modulates activities of glioma cells by targeting HMGB1. Am J Transl Res 8: 3780-3790, 2016.

27. Liu Z, Dou C, Yao B, Xu M, Ding L, Wang Y, Jia Y, Li Q, Zhang $\mathrm{H}, \mathrm{Tu} \mathrm{K}$, et al: Methylation-mediated repression of microRNA-129-2 suppresses cell aggressiveness by inhibiting high mobility group box 1 in human hepatocellular carcinoma. Oncotarget 7: 36909-36923, 2016.

28. Yang J, Chen L, Ding J, Fan Z, Li S, Wu H, Zhang J, Yang C, Wang $\mathrm{H}$, Zeng $\mathrm{P}$ and Yang J: MicroRNA-24 inhibits high glucose-induced vascular smooth muscle cell proliferation and migration by targeting HMGB1. Gene 586: 268-273, 2016.

29. Zhu H, Huang L, Zhu S, Li X, Li Z, Yu C and Yu X: Regulation of autophagy by systemic admission of microRNA-141 to target HMGB1 in 1-arginine-induced acute pancreatitis in vivo. Pancreatology 16: 337-346, 2016. 